Wien klin Mag 2021 · 24:129

https://doi.org/10.1007/s00740-021-00409-w

Angenommen: 16. August 2021

(C) Springer-Verlag GmbH Austria, ein Teil von Springer Nature 2021

Ein direkter Begleiteffekt der umfassenden Digitalisierung ist die zunehmende Vernetzung von Prozessen, von Orten, Personen und Einrichtungen. Das klappt mal besser und mal schlechter. Noch immer stellen Schnittstellen vielerorts ein Problem dar und müssen Daten mehrmals erfasst bzw. analog weitergegeben werden. Das kostet viel Zeit und Nerven und birgt das Risiko des Daten- bzw. Informationsverlusts. Immerhin ist man sich des Problems, besonders auch im Gesundheitswesen, mittlerweile in vielen Bereichen bewusst.

\section{Mangelndes Bewusstsein für Hackerangriffe}

Wenn die Vernetzung allerdings tatsächlich funktioniert, bedeutet dies auch, dass alle beteiligten Nutzer sich des Risikos von weitreichenden unerwünschten Einflussnahmen im System - vulgo Hackerangriffen - bewusst sein sollten. Hier bestehen auf allen Ebenen deutliche Defizite: Von der Management- und Führungsebene bis zum Assistenzpersonal in den medizinischen ebenso wie in den administrativen Abteilungen. Trotz unterschiedlichster Versuche, die Teilnehmer im internen und externen elektronischen Informationsfluss im richtigen Umgang mit Nachrichten und Aufforderungen $\mathrm{zu}$ schulen, ist das sorglose bis naive Öffnen von mitgeschickten Dateien oder angegebenen Verlinkungen nach wie vor der häufigste Fehler. Täuschend ähnlich gestaltete Nachrichten wie jene von Banken, Kunden und anderen Unternehmen suggerieren Seriosität - und sind erstaunlicherweise immer wieder erfolgreich in der Irreführung. Oft müsste man sich nur die E-Mail-Adresse des Absenders genauer anschauen, um den Betrug

Verena Kienast

SpringerMedizin, Wien, Österreich

\title{
Oft ziemlich sorglos
}

\section{Fluch und Segen der Vernetzung}

aufzudecken - und Schaden abzuwenden. Denn die Folgen sind oft drastisch. Lahmgelegte Systeme sind nicht nur ärgerlich und teuer, sondern speziell im Gesundheitsbereich auch lebensbedrohlich.

\section{Gar nicht daran gedacht}

So ergab eine Studie einer Forschergruppe an der FH Münster zum Thema IT-Sicherheit im Klinikalltag deutliche Schwachstellen. Dabei ging es um vernetzte medizinische Geräte und Systeme und hier zeigte sich, dass vielen Mitarbeitern nicht bewusst ist, wie ein Gerät vom anderen abhängig ist und was der Ausfall eines Geräts für die anderen bedeutet. Entsprechende Notfallsysteme sollten hier dafür sorgen, dass innerhalb einer maximal tolerierbaren Ausfallzeit für eine Wiederherstellung des Betriebs gesorgt wird. Ein in einer qualitativen Pilotstudie simulierter, exemplarischer Hackerangriff auf Herzmonitore, die kontrolliert verrücktspielten, wurde von den meisten der Probanden nicht als solcher wahrgenommen, weil sie an diese Möglichkeit gar nicht dachten. Das Bewusstsein dafür sollte deshalb schon in der medizinischen Ausbildung ein wichtiges Thema darstellen, stellen die Forscher fest.

\section{Cyberkompetenz in der Schule vermitteln}

Tatsächlich sollten die Aufmerksamkeit und die Kompetenz in Sachen Cybersicherheit schon viel früher, nämlich in der Schule vermittelt werden. Der Umgang mit elektronischen Medien, die Nutzung des World Wide Web mit seinen unüberschaubaren und perma- nent wachsenden Möglichkeiten ist für die jungen Generationen zur Selbstverständlichkeit geworden, Grundregeln des Verhaltens in dieser virtuellen Welt sind es - noch - nicht. So, wie Kinder schon sehr früh lernen, dass sie besser nicht auf der stark befahrenen Autobahn spazieren gehen oder ohne Schwimmkenntnisse und Schwimmhilfe in tiefes Wasser springen, so sollten sie auch die Grundsicherheitskenntnisse über den Cyberraum als Grundkompetenzen lernen. Und vielleicht können sie dann auch ihren Eltern in Sachen Cybersicherheit noch etwas beibringen.

Meint Ihre

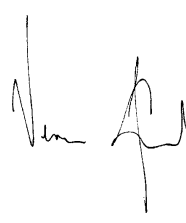

\section{Korrespondenzadresse}

Verena Kienast

SpringerMedizin

Wien, Österreich

Verena.kienast@springer.at

Hinweis des Verlags. Der Verlag bleibt in Hinblick auf geografische Zuordnungen und Gebietsbezeichnungen in veröffentlichten Karten und Institutsadressen neutral. 